\title{
Simulation-Based Airframe Noise Prediction of a Full-Scale, Full Aircraft
}

\author{
Mehdi R. Khorrami* \\ NASA Langley Research Center, Hampton, Virginia, 23681 \\ and \\ Ehab Fares ${ }^{\dagger}$ \\ Exa GmbH, Curiestrasse 4, D-70563 Stuttgart, Germany
}

\begin{abstract}
A previously validated computational approach applied to an $18 \%$-scale, semi-span Gulfstream aircraft model was extended to the full-scale, full-span aircraft in the present investigation. The full-scale flap and main landing gear geometries used in the simulations are nearly identical to those flown on the actual aircraft. The lattice Boltzmann solver PowerFLOW ${ }^{\circledR}$ was used to perform time-accurate predictions of the flow field associated with this aircraft. The simulations were performed at a Mach number of 0.2 with the flap deflected $39^{\circ}$ and main landing gear deployed (landing configuration). Special attention was paid to the accurate prediction of major sources of flap tip and main landing gear noise. Computed farfield noise spectra for three selected baseline configurations (flap deflected $39^{\circ}$ with and without main gear extended, and flap deflected $0^{\circ}$ with gear deployed) are presented. The flap brackets are shown to be important contributors to the farfield noise spectra in the mid- to high-frequency range. Simulated farfield noise spectra for the baseline configurations, obtained using a Ffowcs Williams and Hawkings acoustic analogy approach, were found to be in close agreement with acoustic measurements acquired during the 2006 NASA-Gulfstream joint flight test of the same aircraft.
\end{abstract}

\section{Introduction}

Alleviating the adverse environmental impact of aircraft noise on population centers near airports is a high priority goal of the NASA Aeronautics Research Mission Directorate. As air travel is projected to grow steadily within the next two decades, ${ }^{\ddagger}$ novel concepts that will make next generation civil transports exceptionally quiet must be introduced. Airframe noise is a prominent component of aircraft noise during landing. Wing high-lift devices, such as slats and flaps, and the aircraft undercarriage system are major sources of airframe noise. ${ }^{1-6}$ The NASA Environmentally Responsible Aviation (ERA) project aims to advance and accelerate the development of simulation-based, systemlevel airframe noise prediction tools. Maturation of such tools will help promote a leap in aircraft design from the current time-consuming and costly "cut-and-try" approach to a physics-based, virtual design environment where aeroacoustic evaluation/optimization of a noise reduction concept can take place in an integrated fashion.

Computational simulations have made significant inroads as a viable, complementary tool to wind tunnel testing for airframe noise prediction. Until recently, these complex, high-fidelity simulations were mostly confined to sub- or full-scale airframe components. ${ }^{711}$ As part of the NASA-Gulfstream partnership on airframe noise, time-accurate aeroacoustic simulations were performed on an $18 \%$-scale, semi-span, high-fidelity model of a Gulfstream aircraft with and without flap and landing gear noise mitigation concepts installed. ${ }^{12-15}$ These model-scale simulations represented a first attempt at the prediction of 1) system-level airframe noise for an aircraft in landing configuration and 2) aeroacoustic performance of numerous airframe noise mitigation concepts. Very good agreement between simulated results and wind tunnel measurements of steady and unsteady surface pressure fields, off-surface velocity field, and farfield acoustic behavior of the $18 \%$-scale aircraft model was demonstrated, establishing the validity of the computational approach outlined in Refs. 13-16 as a powerful predictive tool.

The present study is part of a comprehensive computational effort designed to advance the state-of-the-art in airframe noise prediction from sub-scale models to system-level, full-aircraft configurations. Following our earlier model-scale simulations, accurate prediction of the broadband component of airframe noise for full-scale, full-aircraft was attempted via application of a Lattice Boltzmann-Very Large Eddy Simulation (LB-VLES) approach within Exa Corporation's PowerFLOW ${ }^{\circledR}$ solver. Results for several baseline aircraft configurations are presented in this paper.

\footnotetext{
* Aerospace Engineer, Computational AeroSciences Branch, Associate Fellow AIAA

$\dagger$ Technical Director, Aerospace Applications, Senior Member AIAA

$\sharp$ https://www.faa.gov/data_research/aviation/aerospace forecasts, cited November 8, 2015
} 
The same aircraft geometry and computational methodology used in the present study were also applied to a systemlevel, full-scale evaluation of flap and landing gear noise reduction concepts. That effort is summarized in a companion paper by Khorrami et al. ${ }^{17}$

\section{Full-Scale Aircraft Geometry}

The simulated geometry corresponds to a Gulfstream aircraft that was used during the NASA-Gulfstream joint airframe noise flight test conducted in $2006 .{ }^{18} \mathrm{An} 18 \%$-scale, semi-span, high-fidelity reproduction of the same aircraft was used for an extensive study of flap and landing gear noise sources and corresponding noise mitigation concepts. ${ }^{13-}$ ${ }^{14}$ The geometry used for the present work was developed using a full-scale version of the $18 \%$ scale model fuselage, wing, and flow-through nacelle; the flaps, including their complex bracket/track system, as well as the vertical and horizontal tails, were developed using as-flown, full-scale geometry files furnished by Gulfstream. The main landing gear, including the wheel cavity, was developed from the original full-scale geometry files containing the finer details of the gear as deployed on the actual aircraft. The larger structures residing within the wheel well that could potentially alter the cavity volume were also included. Because the gear cavity extends across the fuselage, the full aircraft span had to be considered in the simulations. The computational cost and resources required for each aircraft configuration to be simulated were significantly higher than those used for the $18 \%$ scale, semi-span model simulations ${ }^{13,14}$ mainly due to the increased Re and inclusion of the full-span geometry.

For the purpose of eventually evaluating the aeroacoustic performance of a select number of flap and gear noise abatement technologies, three aircraft configurations were identified as baselines. They correspond to three of the four configurations evaluated during the 2006 flight test (see Fig. 1) and served as baselines for evaluating the aeroacoustic performance of numerous flap and gear noise abatement concepts during the $18 \%$-scale model test. The configuration with the flaps deflected $39^{\circ}$ and main gear retracted (Fig. 1a) was used to isolate the noise sources associated with the flap and to determine the simulated, system-level, aeroacoustic performance of a select number of flap tip treatments. ${ }^{14,19}$ The second configuration with the flaps at $0^{\circ}$ (cruise wing) and main gear deployed (Fig. 1b), although not a landing condition, was used to isolate the noise sources associated with the main landing gear and to determine the effectiveness of several gear fairings in reducing the noise from this component. ${ }^{14}$ The configuration with $39^{\circ}$ flap deflection and main landing gear deployed (Fig. 1c) represents a more complete aircraft geometry during landing and was used to determine the efficacy of the flap and gear noise mitigation technologies when applied concurrently. ${ }^{14,19}$
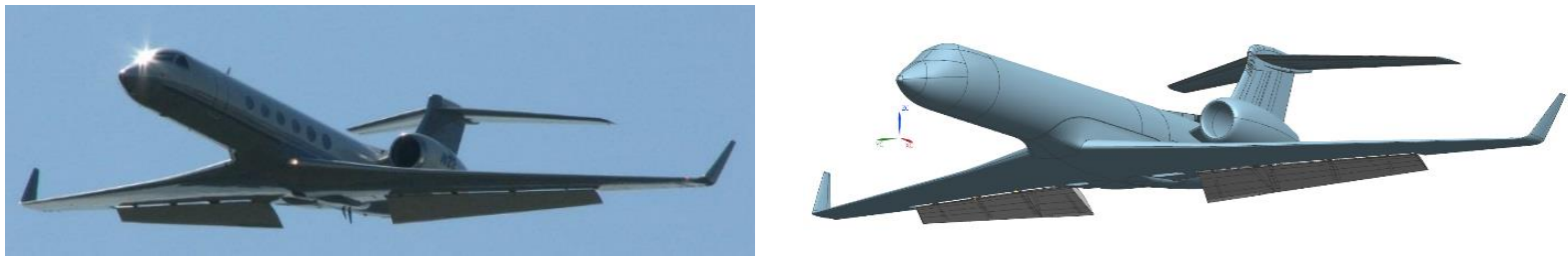

a) $39^{\circ}$ deflected flap with main gear retracted, left image 2006 flight test, right image simulated CAD model
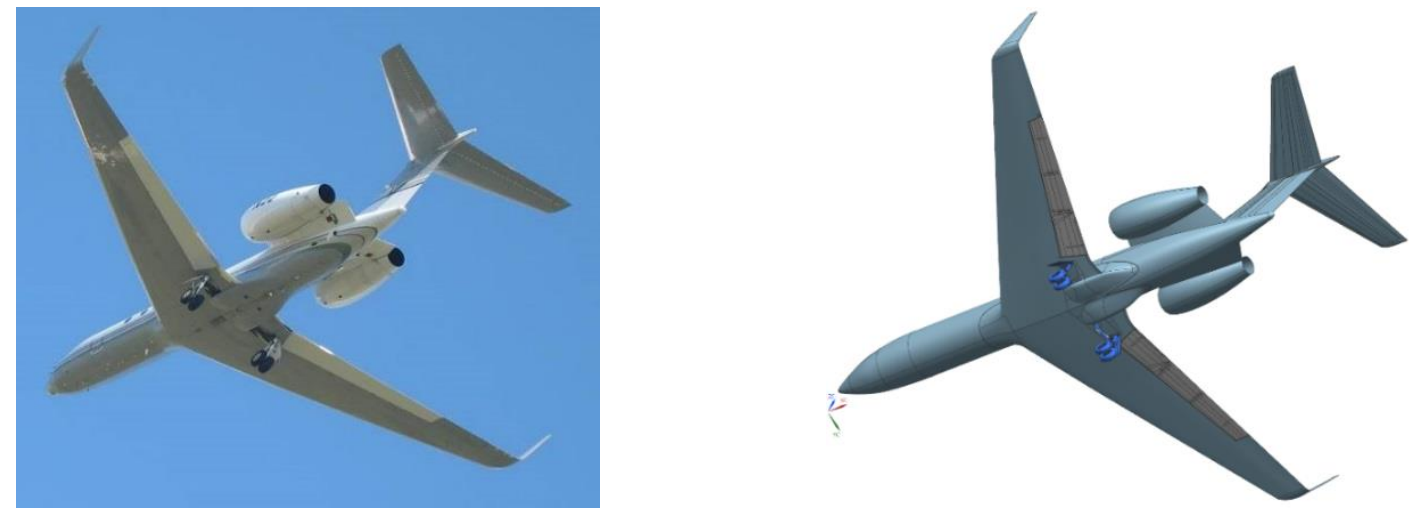

b) $0^{\circ}$ flap (cruise wing) with main gear deployed, left image 2006 flight test, right image simulated CAD model

Figure 1. Baseline configurations for full scale Gulfstream aircraft (continued). 

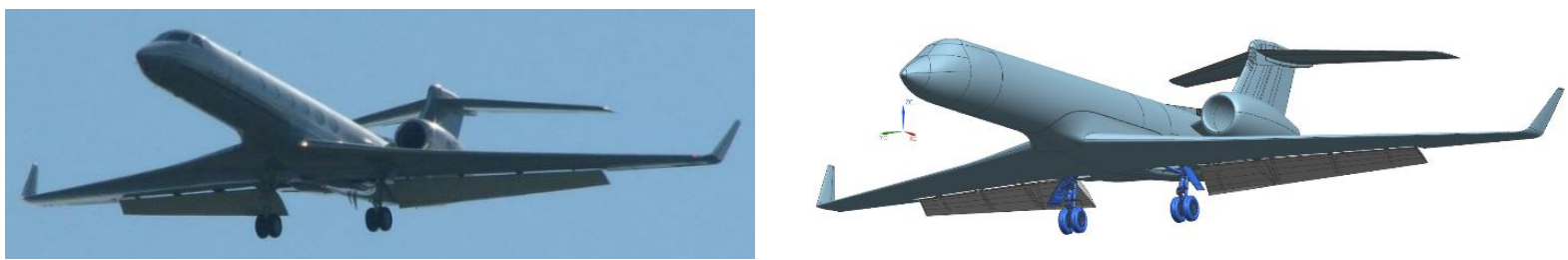

c) $39^{\circ}$ deflected flap with main gear deployed, left image 2006 flight test, right image simulated CAD model

Figure 1. Concluded.

\section{Computational Approach}

The computational approach used in the present effort is a direct extension of the methodology outlined in Refs. 13-14 to an entire full-scale aircraft. The numerical simulations were performed using the commercial flow solver PowerFLOW $^{\circledR}$, which is based on the three dimensional 19 state (D3Q19) lattice Boltzmann model (LBM). The software has been successfully validated for flows around highly complex airframe geometries such as those reported in Refs. 7-9, 11, and 13-15.

\section{A. Flow Solver}

The lattice Boltzmann equations represent an alternative mesoscopic formulation to the classical macroscopic Navier-Stokes equations describing a compressible unsteady flow of a continuum. The details of the mathematical foundations are documented in Refs. 9, 11 and 20. The simple, local formulation of the underlying D3Q19 equations allows a highly efficient implementation for distributed computations on thousands of processors. The low dissipation and dispersion properties of the numerical scheme typically produce aerodynamic and aeroacoustic results that are comparable to those obtained with classical CFD solvers that use higher-order large eddy simulation (LES), as demonstrated in the comparative study of flow over tandem cylinders presented in Ref. 21.

\section{B. Turbulence Modeling}

The lattice Boltzmann flow simulation is equivalent to a Direct Numerical Simulation (DNS) of the flow. For high Reynolds number flows, such as those addressed in this work, the Lattice Boltzmann Very Large Eddy Simulation (LB-VLES) approach described in Refs. 22 and 23 is used in conjunction with an extended wall function. The approach has been applied previously in Refs. 9, 13, and 14.

\section{Computational Grids}

The lattice Boltzmann formulation is solved on Cartesian meshes that are generated automatically within PowerFLOW® for any geometrically complex shape. This greatly simplifies the labor-intensive volume meshing step usually associated with other approaches. Gridding strategy, overall mesh distribution, and arrangement of the variable resolution (VR) regions for various grids were described in Refs. 13, 14, and 16.

\section{Boundary Conditions}

The simulations were performed for free-air. All aircraft surfaces were modeled with no slip boundary conditions via a generalized bounce back volumetric formulation ${ }^{24,25}$ near the wall for arbitrarily oriented surfels within the voxels. Far field boundary conditions for velocity, turbulent kinetic energy and pressure were used at the inflow and outflow boundaries. In addition, sponge layer zones were included in the far field to damp reflections of acoustic waves.

\section{E. Computational Procedure}

The PowerFLOW ${ }^{\circledR}$ simulations were initialized with freestream conditions for a period corresponding to the time that it would take the flow to convect a distance of about 30 mean aerodynamic chords. The time steps $(\Delta \mathrm{t})$ used for the current set of simulations were $5.44 \times 10^{-7} \mathrm{~s}, 8.15 \times 10^{-7} \mathrm{~s}$, and $1.22 \times 10^{-6} \mathrm{~s}$ corresponding to the fine, medium, and coarse grids, respectively. Statistical convergence of the flow solution during this initial transient time was monitored using unsteady pressure signals acquired on the surface of the wing and the time history of the global aerodynamic forces acting on the aircraft.

After reaching statistical convergence within the first $2.0 \mathrm{~s}$ of simulated physical time, the simulation was run an additional $1.0 \mathrm{~s}$ of physical time during which the computed flow variables were stored in a number of different volume and surface measurement files for subsequent flow analysis and post-processing. The flow variables were recorded with various sampling frequencies (for the fine simulation) ranging from about $57 \mathrm{kHz}$ for the surface to 15 
$\mathrm{kHz}$ in some areas of the volume. Post-processing spectral analyses were conducted using a bandwidth of $3.6 \mathrm{~Hz}$ and an overlapping coefficient of 0.5 . Results were compared with measured data up to $10 \mathrm{kHz}$.

An acoustic analogy approach based on the Ffowcs Williams and Hawkings (FWH) formulation ${ }^{26}$ was used to extrapolate the computed near-field fluctuations to the far field. The employed FWH formulation was based on the retarded-time formulation $1 \mathrm{~A}$ by Farassat, ${ }^{27}$ extended to account for uniform mean flow convection effects to simulate the noise generated and measured in an ideal infinite wind tunnel. ${ }^{28}$

\section{Results and Discussion}

The computations presented here were executed at $M=0.2$. A Reynolds number (Re) of $10.5 \times 10^{6}$, roughly half the flight Re (based on the full scale wing mean aerodynamic chord and aircraft landing speed), was chosen for the simulations. This particular value was selected after a modest study targeting Reynolds number effects revealed that beyond $\mathrm{Re}=10 \times 10^{6}$ scale effects on steady and unsteady surface pressure fields diminished significantly. ${ }^{16}$ Accordingly, we believe that the farfield noise levels obtained at this Re are nearly equivalent (at a lower computational cost) to the corresponding levels at the full flight Re.

As a first step, solution convergence was examined carefully. Given the resource-intensive nature of the present computations, the convergence study was used to determine the most efficient VR setup and the minimum required spatial resolution that would yield a relatively grid-converged solution within a reasonable computational cost. Relying on the experience gained from the $18 \%$-scale model predictions, ${ }^{13}$ simulations on coarse, medium, and fine resolution grids were performed. The global VR setup and mesh refinement strategy developed for the sub-scale computations was adopted to enable similar resolutions for the boundary and shear layers at the increased Re of $10.5 \times 10^{6}$. A summary of the computational attributes and resources for the largest case (flap $39^{\circ}$ and main gear deployed) is presented in Table 1.

Table 1. Simulation statistics.

\begin{tabular}{|c|c|c|c|}
\hline $\begin{array}{c}\text { Baseline } \\
\text { Full span }- \text { full scale }\end{array}$ & Coarse resolution & Medium resolution & Fine resolution \\
\hline Minimum cell size $[\mathrm{mm}]$ & 0.745 & 0.497 & 0.331 \\
\hline Volume elements $\left[10^{6}\right]$ & 896 & 2,609 & 8,396 \\
\hline Surface elements $\left[10^{6}\right]$ & 62 & 108 & 287 \\
\hline Simulated time $[\mathrm{s}]$ & 3.0 & 3.0 & 3.0 \\
\hline Time steps & $2,453,077$ & $3,679,615$ & $5,519,422$ \\
\hline Cores used for computation & 2,000 Ivy & 4,020 Westmere & 12,000 Ivy \\
\hline Surface sampling frequency & 25,551 & 38,327 & 57,490 \\
\hline CPU] $\left[10^{3}\right]$ & 190 & 991 & $\sim 7,000$ \\
\hline
\end{tabular}

Sample results of steady and unsteady surface pressures from the baseline configuration of $39^{\circ}$ deflected flaps with the main landing gear on (deployed), which represents the most computationally demanding configuration, are presented next to highlight some of the more pertinent trends. The locations of the steady pressure rows and unsteady pressure probes and the naming convention used to identify them are the same as those used for the $18 \%$ scale model study. ${ }^{12,13}$ For completeness, they are reproduced in Figs. 2 and 3. 


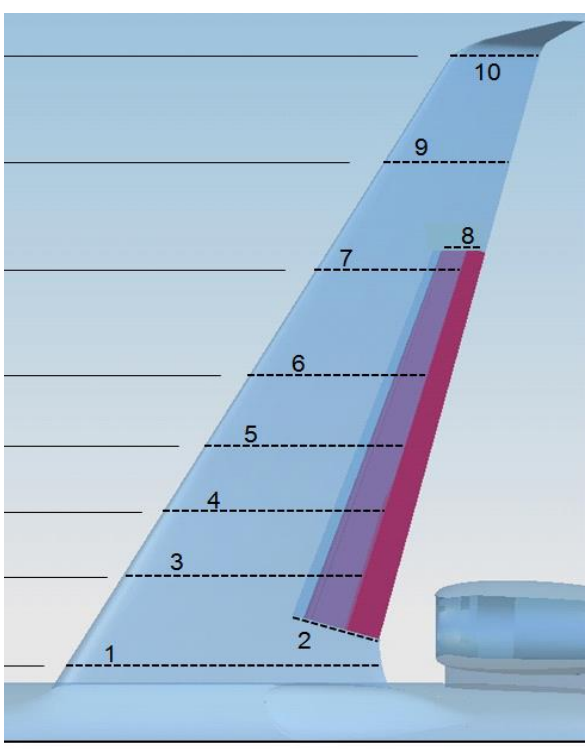

a) Wing

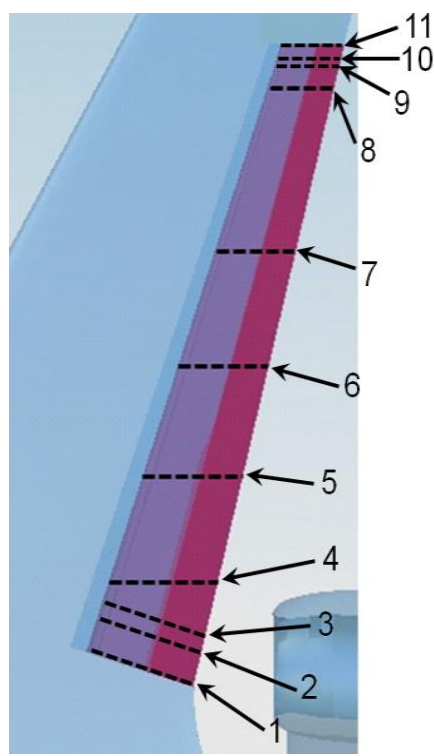

b) Flap

Figure 2. Spanwise location and numbering of steady pressure rows used on $18 \%$ scale model wing and flap.

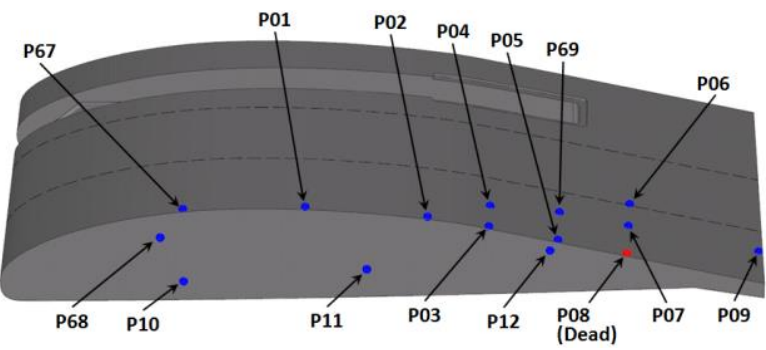

a) Inboard tip

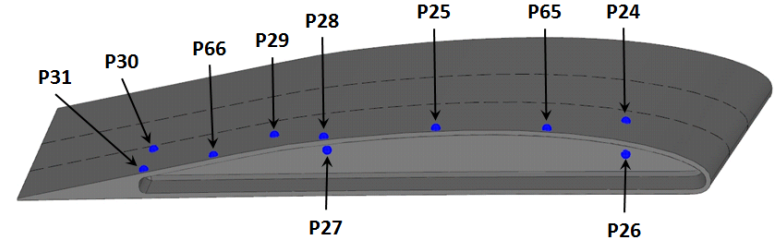

b) Outboard tip

Figure 3. Location and numbering of unsteady pressure transducers used on $18 \%$ scale model flap tips.

\section{A. Solution Dependency on Mesh Resolution}

Steady surface pressure distribution on the wing at a spanwise location corresponding to the flap mid-span position (row 5) is shown in Fig. 4. Results from coarse, medium, and fine grids are shown in black, blue, and red symbols, respectively. The extracted pressure coefficients $(\mathrm{Cp})$ from the three grids are nearly identical, depicting a well converged wing loading even at the medium-resolution level. Similar convergence behavior was obtained at other pressure rows on the wing (not shown). For accurate computation of flap noise, prediction of the flap loading, in particular in the vicinity of the inboard and outboard tips, is of paramount importance. The chordwise pressure distributions at flap mid-span location (row 6), inboard (row 1), and outboard (row 11) side edges are shown in Figs. 4b-d. At the midspan and inboard edge, the steady pressure field is well converged and only minor differences among the solutions obtained from the three grids are observed. At the outboard edge (Fig. 4d), however, while the overall pressure distribution trends are maintained, the magnitude of the pressure coefficient on the flap suction side shows noticeable differences with grid resolution. Notice that solution convergence is not monotonic since the coarsest results reside between the medium and fine resolution solutions. We attribute this behavior to the highly complex, non-linear interaction that occurs between rolling up of the free shear layer and the flow field associated with the tip cavity housing the bulb seal. This interaction causes jitter in the spanwise position of the tip vortex resulting from the rolled up shear layer, thus making the time-averaged spanwise vortex location extremely sensitive to grid refinement. 


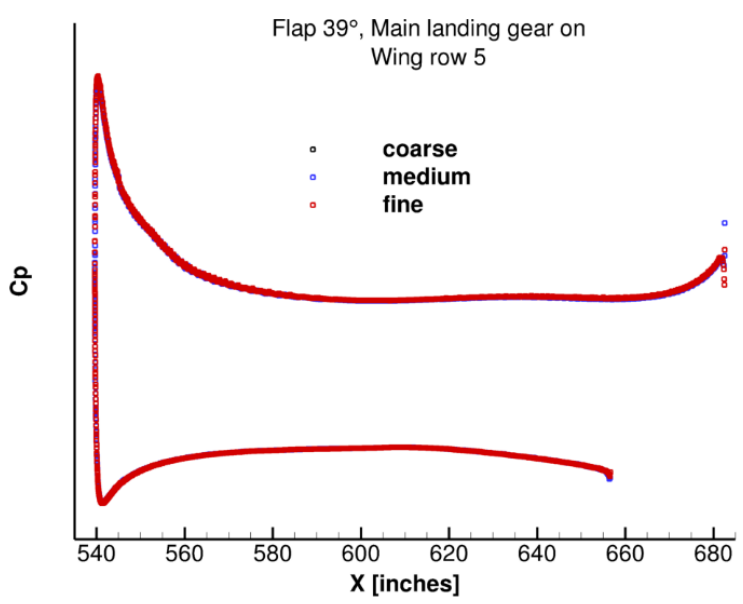

a) Wing

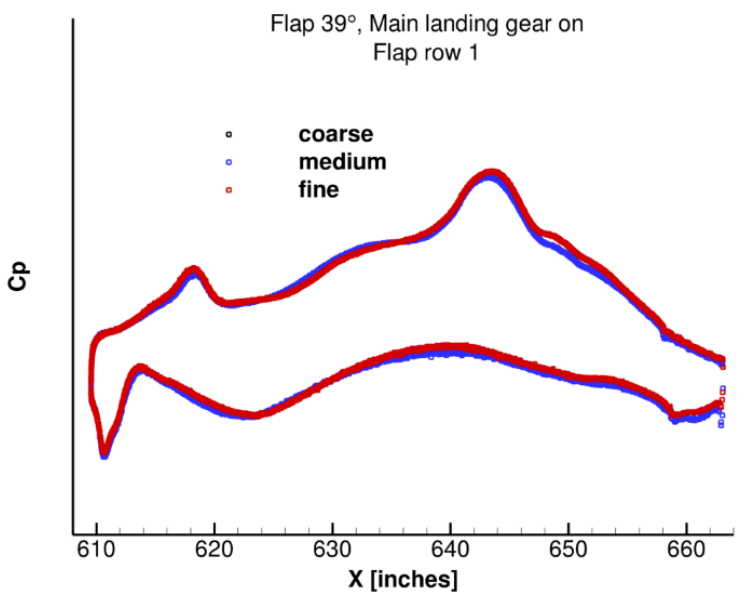

c) Flap inboard edge

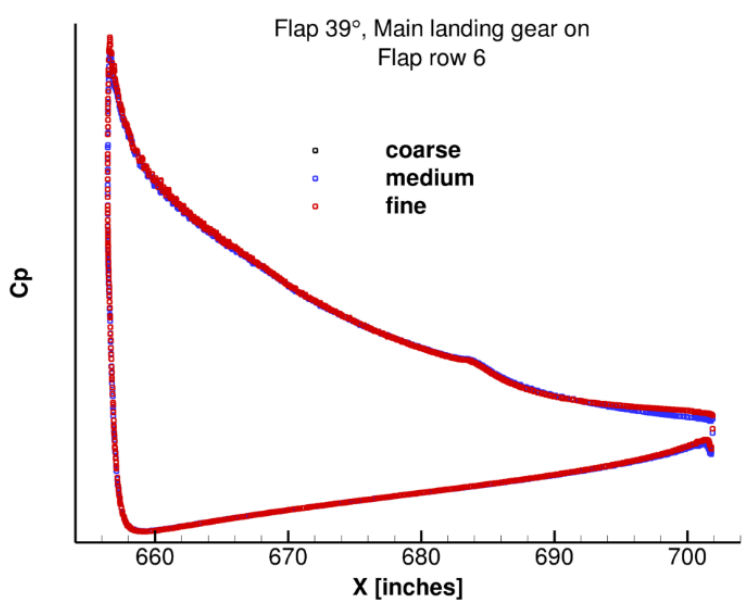

b) Mid-span flap

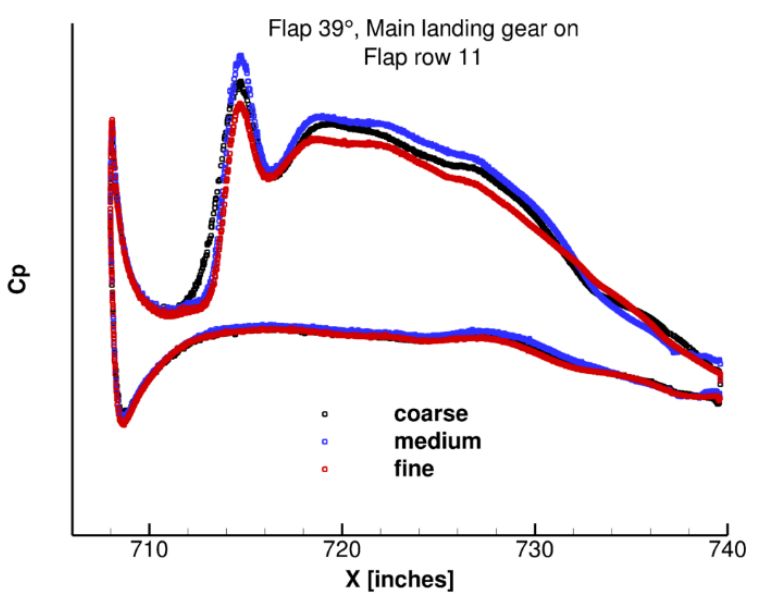

d) Flap outboard edge

Figure 4. Steady pressure distributions on aircraft wing and flap.

An equally important aspect of solution quality is convergence of the surface pressure fluctuations. As sample results, we have picked two probe locations at each flap tip to discuss resolution effects on the time-dependent pressure field. The power spectral density (PSD) data for the two probes located at the inboard tip, one positioned near the edge (probe 3 ) and one away from the edge (probe 6), are plotted in Fig. 5a. A nearly grid-independent solution was achieved. We remark here that solution convergence at other probe locations on the inboard tip is similar with some probes showing slightly better and some probes slightly worse results than those shown in Fig. 5a. PSD results for the two selected probes at the outboard tip are displayed in Fig. 5b. The surface pressure spectra collected at probe 27, which is located inside the tip cavity, show a good collapse of the data obtained from the three grids indicating that the cavity flow field is nicely resolved even at the medium resolution level. In contrast, the spectra at probe 30 , positioned away from the edge under the path of the primary tip vortex, indicate a significant variation with mesh resolution. As observed for the steady pressure distribution at the outboard tip (Fig. 4d), the convergence behavior of the PSD levels is not monotonic in nature. In fact, even the lower frequency content of the spectra displays a gradual movement towards a mesh independent solution. As mentioned earlier, we believe such behavior is caused primarily by low frequency variations in vortex position relative to the side edge as the grid resolution is increased. A small sideways movement of the tip vortex could potentially instigate disproportionately large variations in the local fluctuating surface pressures at the same location, very much like those displayed for probe 30 . In general, solution dependency on spatial resolution at most probe locations examined is rather small, with behavior similar to that shown for probe 27 (Fig. 5b). Thus, noticeable differences in PSD levels at a few discrete points should not be used to judge solution accuracy. 

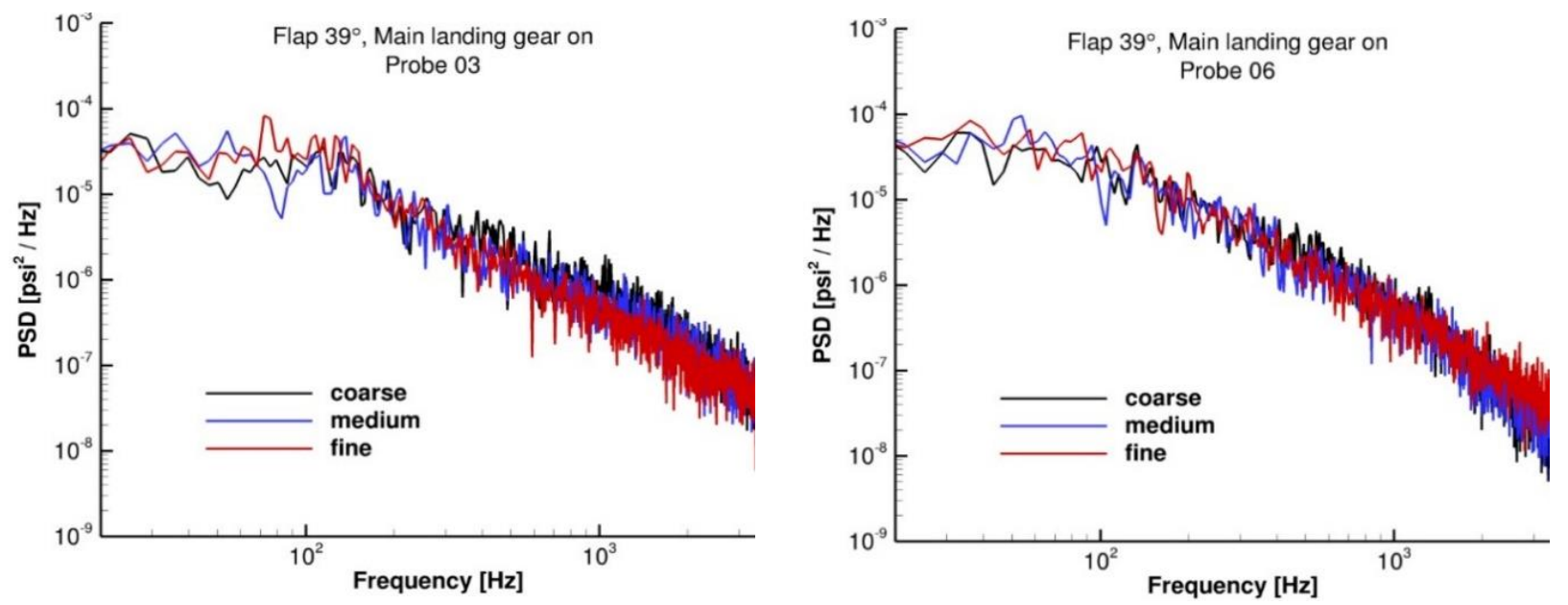

a) Inboard tip
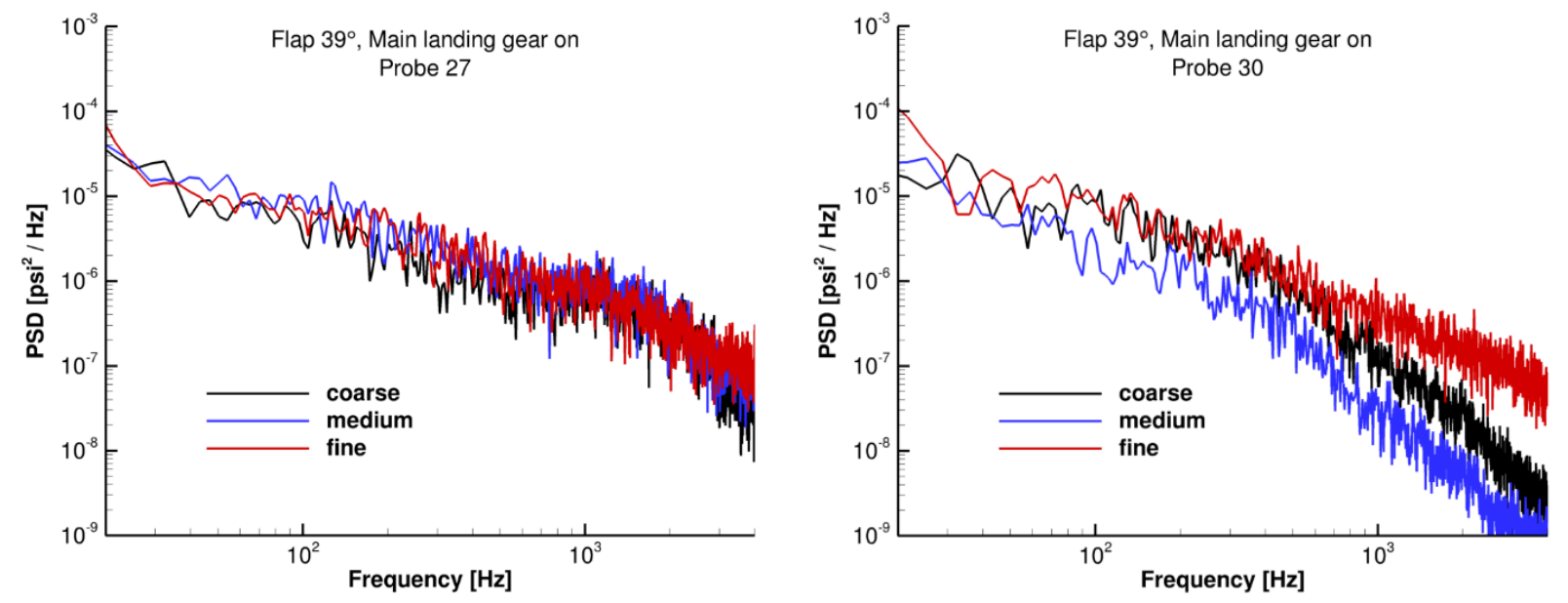

b) Outboard tip

Figure 5. Fluctuating surface pressure field at select locations near flap tips.

\section{B. Validation of Surface Pressures}

As a key component of the NASA-Gulfstream partnership effort, an aerodynamic flight test of the same aircraft was conducted by GAC during March of 2013. For this test, critical elements of the aircraft (e.g., flap, wing, main gear, etc.) were instrumented so that steady and unsteady surface pressures could be collected at the same locations as those measured on the $18 \%$ scale semi-span model. An initial, cursory comparison of the (uncorrected) flight data with the measurements obtained in the $14 \times 22$ tunnel for the $18 \%$-scale model revealed good correspondence between the two sets of data. However, analysis of the surface pressures from the flight test is ongoing at GAC and a fully vetted set of results that could be used for validating the present simulations has not been publicly released yet. The only fully vetted, published aerodynamic measurements are those associated with $18 \%$-scale, semi-span model tests in the LaRC $14 \times 22$ tunnel. ${ }^{29,30}$ Although we expect some differences due to Re and geometry effects to emerge ${ }^{16}$ in regions where significant flow unsteadiness exist (i.e., flap tips, main gear, etc.), comparison of simulated full-scale surface pressures with those measured for the $18 \%$-scale model provides valuable insights on the accuracy of the present simulations. For the sake of completeness, the previously obtained and validated model scale computational results ${ }^{13}$ have also been included in the comparative plots that follow. In these plots, the model scale data have been converted to full scale values using appropriate scaling factors.

Sample plots comparing computed full-scale steady pressures with measured model scale values are presented in Fig. 6. Note from Fig. 6a that the pressure distribution on the wing remains nearly the same except for minor increments in suction at the leading edge and towards the trailing edge segment of the wing. Since the full-scale wing is an enlarged replica of the model-scale wing, the small changes observed are purely caused by Re effects. The plots for 
the steady pressures on the flap also indicate that a close correspondence exists between the full- and model-scale data, especially at the mid-span and inboard tip rows. The most noticeable differences occur at row 11, which is situated adjacent to the flap outboard edge. At this location, the observed increase in pressure on the top and bottom surfaces is due to Re effects and the added geometrical complexities associated with the full-scale tip cavity and bulb seal assembly. ${ }^{16}$ Separation of these effects is not a simple matter. Despite the differences, the overall character of the outboard tip loading generally follows the trends observed for the $18 \%$-scale model.

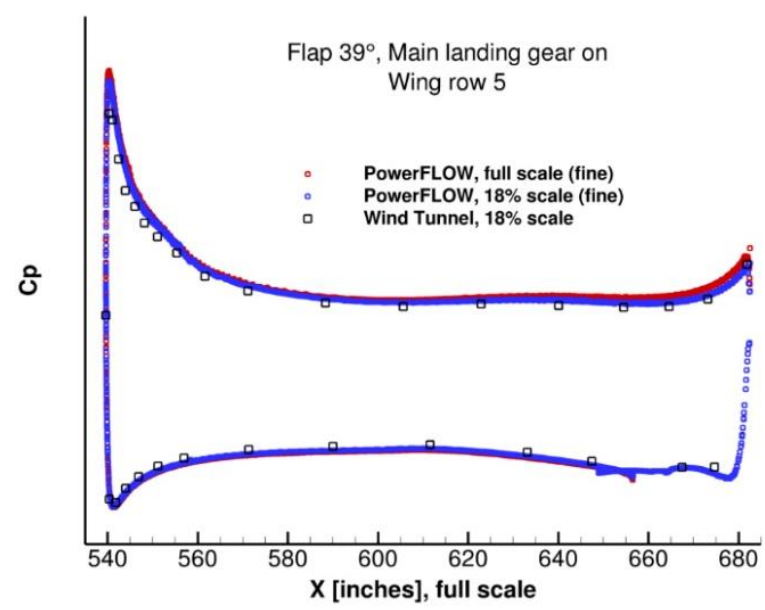

a) Wing

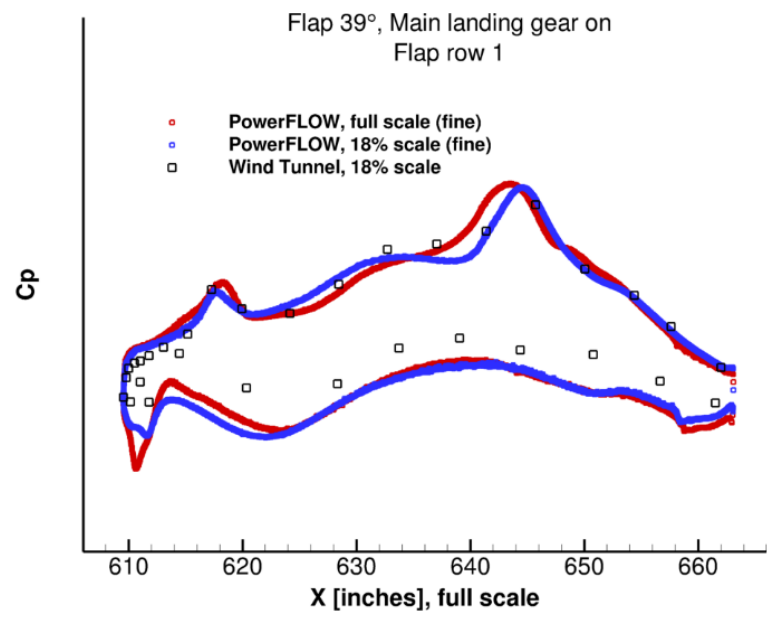

c) Flap inboard edge

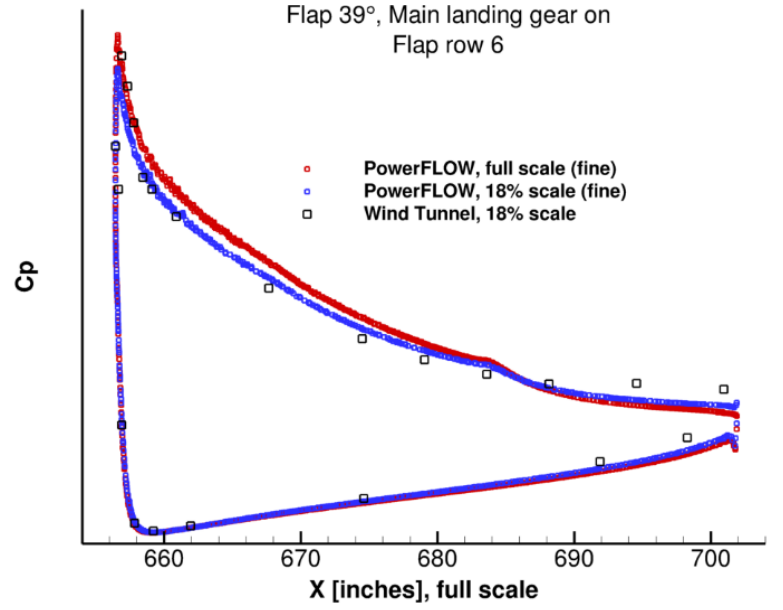

b) Mid-span flap

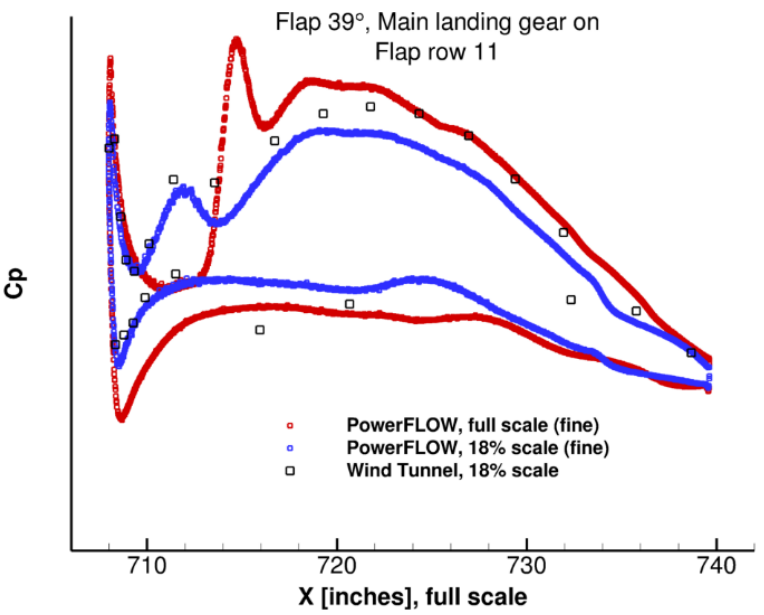

d) Flap outboard edge

Figure 6. Steady pressure distributions on aircraft wing and flap.

Sample comparison plots for the fluctuating surface pressures are given in Fig. 7. For consistency, PSD results are provided at the same probe locations as those used to discuss solution convergence. At the flap inboard tip (probes 3 and 6, Fig. 7a), the extracted full-scale spectra are in close agreement with both measured and simulated model-scale data. In general, for most probes located near the inboard tip, good agreement between the computed full-scale spectra and model-scale measurements is observed. Unsteady pressure spectra at the outboard tip are shown in Fig. 7b. As anticipated, noticeable differences between full-scale, simulated spectra and wing tunnel measurements are observed. We note that similar differences also exist between measured and simulated model-scale results. As was the case for the steady pressure distribution in the outboard tip region, inherent Re and geometry effects associated with the fullscale spectra are the main causes of the observed differences. However, the overall character and trend of the simulated fluctuating pressure field at the outboard tip are in agreement with those observed for the $18 \%$-scale model. 

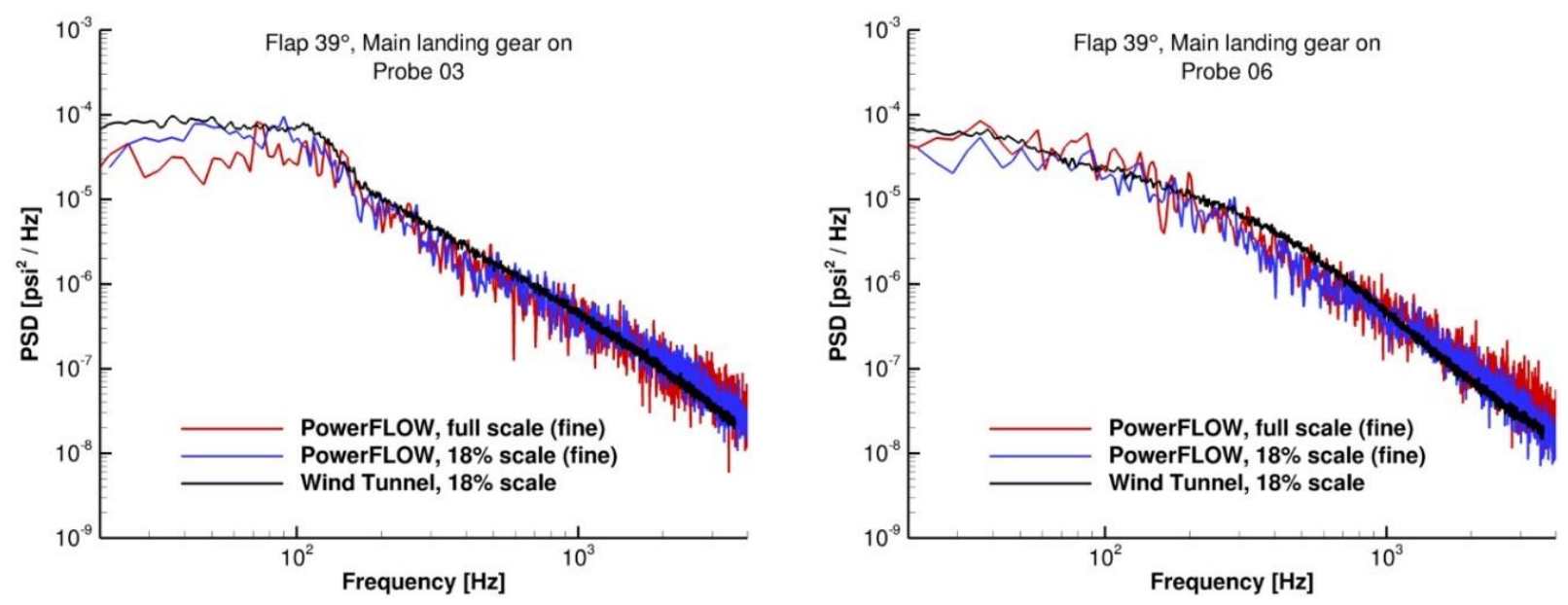

a) Inboard tip
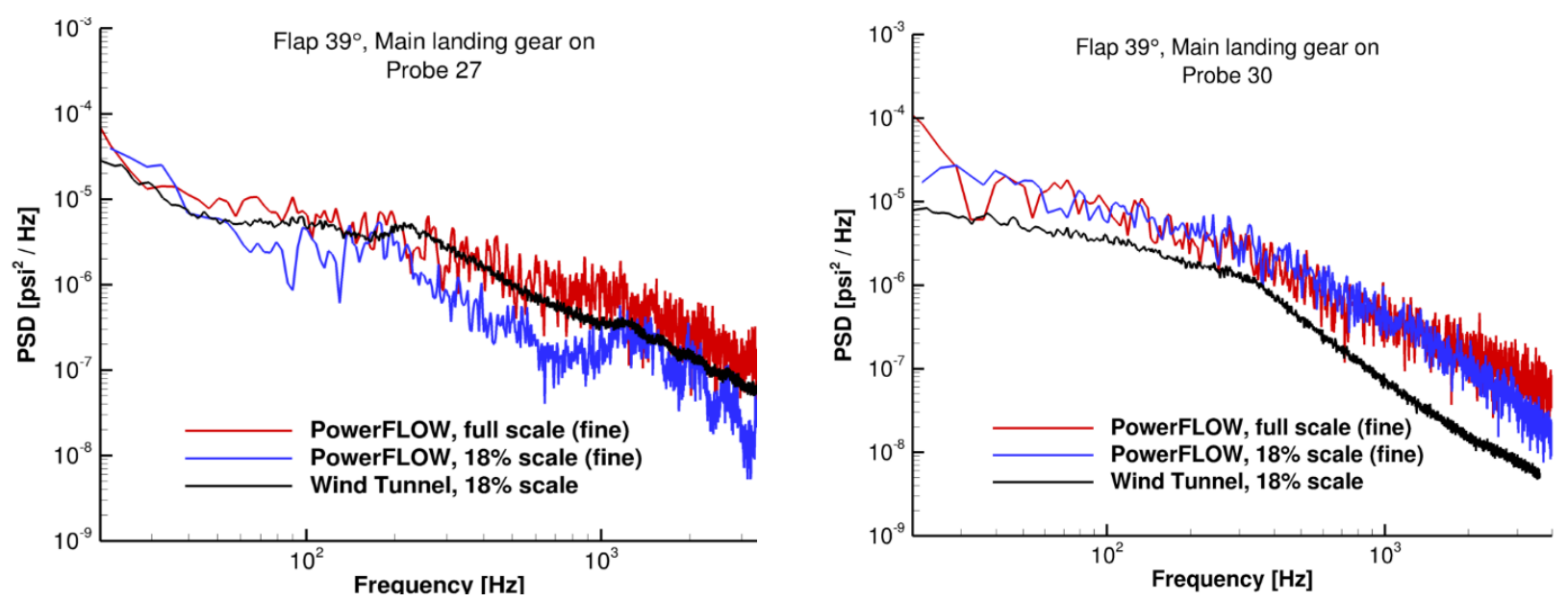

b) Outboard tip

Figure 7. Unsteady surface pressures at select locations near flap tips.

Although not shown, comparison of the unsteady pressure field measured on the main landing gear shows similar trends to those observed at the flap outboard tip. That is, while good correspondence between full-scale and modelscale spectra is observed at most probe locations, considerable differences emerge at some positions. This behavior was fully expected as the full-scale main gear contains finer geometrical details that were absent in the $18 \%$-scale model that was tested and simulated. In addition, Re effects also play an important role in altering the highly nonlinear flow field associated with the multitude of bluff bodies that constitute such a complex gear.

\section{Global View}

A global picture of the simulated vorticity field for the landing configuration of flaps deflected $39^{\circ}$ and main gear deployed is presented in Fig. 8. As mentioned earlier, this configuration is the most complex and computationally intensive of the three baselines. The figure clearly depicts where the most prominent flow unsteadiness occur. As expected, the flap inboard and outboard tips, as well as the main landing gear, produce high-amplitude flow fluctuations. Also prominent in this figure is the level of flow unsteadiness that is generated by the flap brackets.

A global view of the radiated sound field produced by this aircraft configuration is given in Fig. 9. The twodimensional planar cut, positioned near the flap leading edge, clearly shows the origin of the stronger sound waves as they radiate spherically from both inboard and outboard flap tips. Close proximity of the main landing gear to the inboard flap tip precludes separation of the noise generated by each component. Although less noticeable than the noise originating from the flap tips, the waves generated by the brackets are clearly discernible. As discussed by Fares et al., ${ }^{16}$ the flap brackets for this aircraft are important secondary sources that contribute a significant amount of noise in the mid- to high-frequency range. 


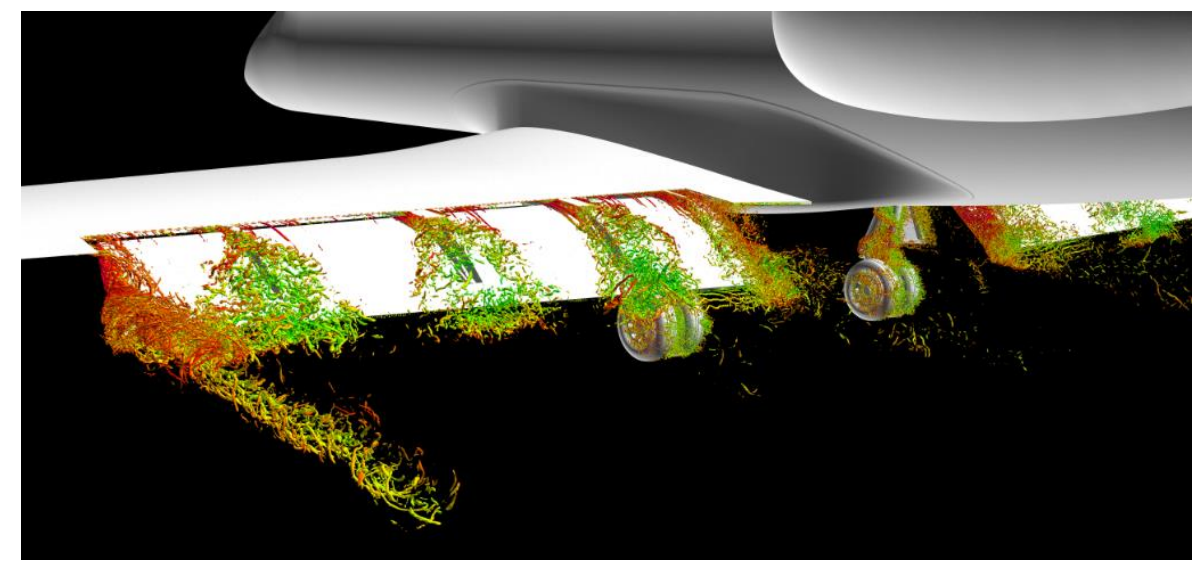

Figure 8. Instantaneous vorticity field based on isosurface of $\lambda_{2}$ criterion for $39^{\circ}$ flap deflection and main gear deployed configuration.

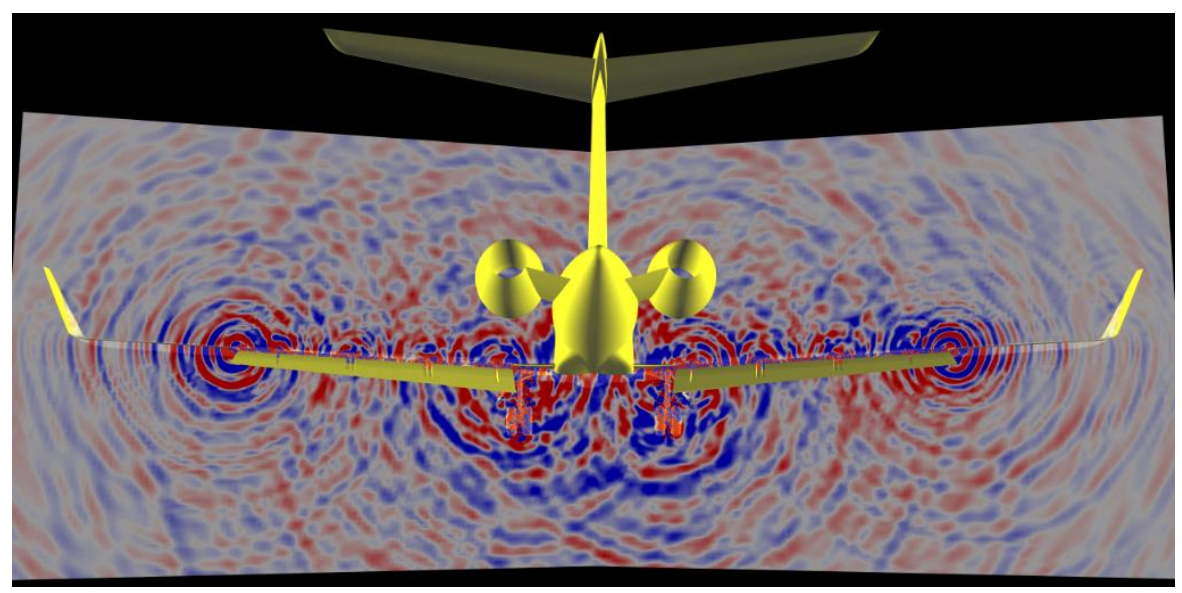

Figure 9. Radiated sound field (dilatation field) associated with baseline configuration for $39^{\circ}$ flap deflection and main gear deployed configuration (planar cut near flap leading edge).

\section{Farfield Noise Signature}

A good portion of flap high-frequency noise was found to be associated with the finer details of the complex fullscale brackets. ${ }^{16}$ Figure 10 shows the $18 \%$-scale model brackets and the corresponding full-scale geometries that were used to design the wind tunnel model. Although the model-scale brackets were carefully reproduced from full-scale design drawings provided by GAC, fabrication and instrumentation limitations necessitated significant geometric simplifications. The current full-scale, full-aircraft simulations are free of such limitations and thus include all the finer bracket details. As can be seen in Fig. 10b, the full-scale brackets contain numerous cut-outs of various shapes and sizes. In particular, they possess many smaller holes and openings. Most of these small holes/openings were the result of missing screws, bolts, and other surface definition mismatches. As discussed in the study of Fares et al., ${ }^{16}$ these smaller holes/openings were not fully resolved in our original simulations and caused excess production of highfrequency noise by the brackets. To partially eliminate bracket high-frequency noise, various relatively small gaps and holes that lacked proper resolution were closed and the resulting configuration is referred to as "modified brackets". Results for farfield noise spectra are shown for both original and modified brackets for those configurations involving deployed flaps. 


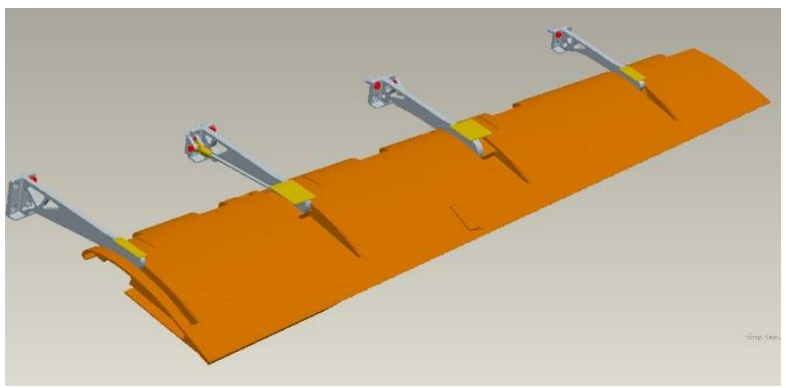

(a) $18 \%$-scale model

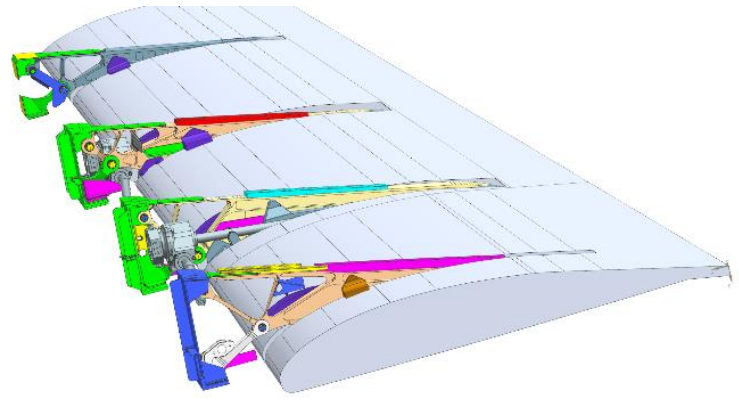

b) Full-scale geometry.

Figure 10. Flap bracket assembly.

The farfield noise computation was conducted using an FWH propagation formulation ${ }^{28}$ on the solid surface of the complete full-scale aircraft. The data sampling frequency for the collected pressure field on the solid surface was 57 $\mathrm{kHz}$. Predicted noise spectra are for the farfield microphone located 394 feet $(120 \mathrm{~m})$ from the aircraft center of rotation, corresponding to the flyover certification point. For comparison with available flight test data, the simulated spectra have been corrected for atmospheric absorption using as reference a standard acoustic day of $25^{\circ}$ Centigrade and $70 \%$ relative humidity. To facilitate direct comparisons with acoustic measurements from the 2006 flight test campaign, two additional corrective steps were taken. The first step accounts for ground reflection effects present in the pole-mounted certification microphone measurements of 2006. For pole microphones located over rigid surfaces (e.g., asphalt, concrete, etc.), the standard correction of adding $3 \mathrm{~dB}$ to the simulated levels applies. However, the pole microphone used during the 2006 flight test was placed over grassy and relatively soft terrain, making proper determination of an amplitude correction a very challenging task. Under such circumstances, the appropriate correction could range anywhere between $1 \mathrm{~dB}$ to nearly $3 \mathrm{~dB}$. Thus, we decided to add $2 \mathrm{~dB}$ to the computed farfield noise levels. Once chosen, the $2 \mathrm{~dB}$ amplitude correction was applied to all subsequent configurations that were simulated without prior knowledge of how the modified spectra would fair against the measured values. The second corrective step involved removal of a well-known tone with peculiar behavior from the flight test spectra for configurations with the main gear deployed. Figure 11a depicts the measured farfield spectrum for the configuration with flap deflected $0^{\circ}$ and main gear deployed. A prominent tone at the $1 / 3^{\text {rd }}$-octave center frequency of $790 \mathrm{~Hz}$ is apparent. A joint study by NASA and Gulfstream (unpublished results) revealed that the tone is produced by a pin cavity present at the knee joint of the main gear front post (see Fig. 11b). Close examination of the beamform maps obtained from the microphone array measurements indicated that, depending on the aircraft speed and the direction of the cross wind, the tone appeared on either the starboard or port side main gear but not on both simultaneously. As such, accurate prediction of this tone in an idealized simulation where local variations in atmospheric conditions (i.e., wind gust, cross wind magnitude and direction, etc.) are not accounted for represented a daunting and unnecessary task since the main goal of the present effort is the accurate prediction of the broadband component of airframe noise. Thus, for validation of the simulated full-scale results, the $790 \mathrm{~Hz}$ tone associated with the pin cavity was replaced in the measured spectra with a value corresponding to the average (on a pressure squared basis) of the levels for the adjacent bands.

One-third octave sound pressure levels (SPL) computed at the $90^{\circ}$ flyover position for the baseline case of $39^{\circ}$ flap deflection with the main gear retracted are shown in Fig. 12. Results from medium and fine resolution simulations for both original and modified brackets are plotted. In general, very good agreement between prediction and certification microphone measurements from the 2006 flight test was attained. Note that most of the differences occur at frequencies above $2 \mathrm{kHz}$, where higher uncertainties caused by local atmospheric effects during the flight test may be present. Also present at high frequencies is residual propulsion noise, even though the engines were operated at near idle conditions during flyover. For frequencies up to $1 \mathrm{kHz}$, the simulated spectra are nearly the same indicating that grid resolution and bracket effects are not impacting the farfield noise signature. Above $1 \mathrm{kHz}$, bracket noise increases gradually with frequency. A drop in spectral levels with increasing grid refinement is observed at higher frequencies for the original bracket configuration. This drop is mostly associated with the elimination or reduction of high frequency tones generated by under-resolved small holes/openings. Also note that better resolution reduces the differences between the noise levels obtained with the original brackets and those associated with the modified brackets. In contrast, grid refinement has a very modest impact on high frequency noise for the modified bracket configuration. The fact that some of the smaller holes/openings that were covered in the simulations could remain open in an actual aircraft precludes a clear determination of bracket noise levels. In our opinion, the high-frequency component of the 
farfield spectra computed for the configurations with flaps deflected should lie between the original and modified bracket levels.

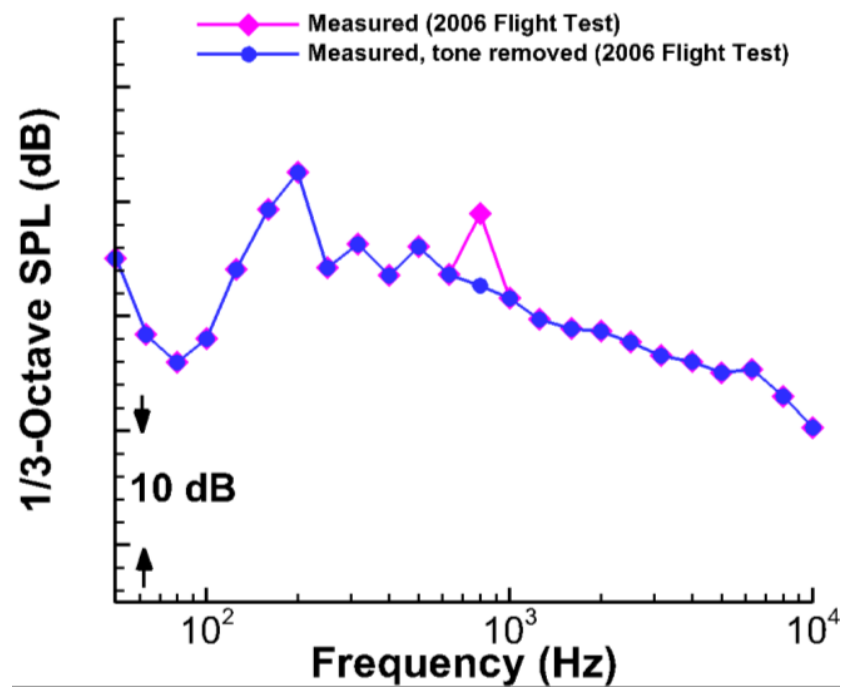

(a) Measured spectrum

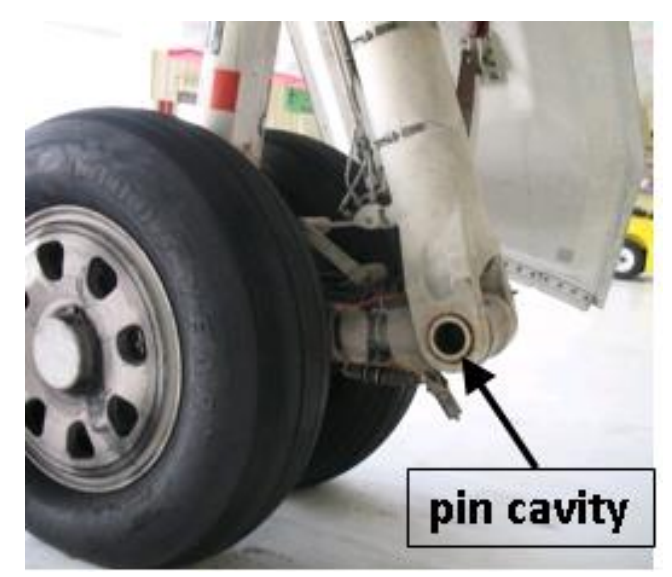

(b) Detail of main gear front post

Figure 11. Measured farfield spectra for configuration with flap $0^{\circ}$ and main gear deployed showing the tone associated with the pin cavity at the knee joint of front post.

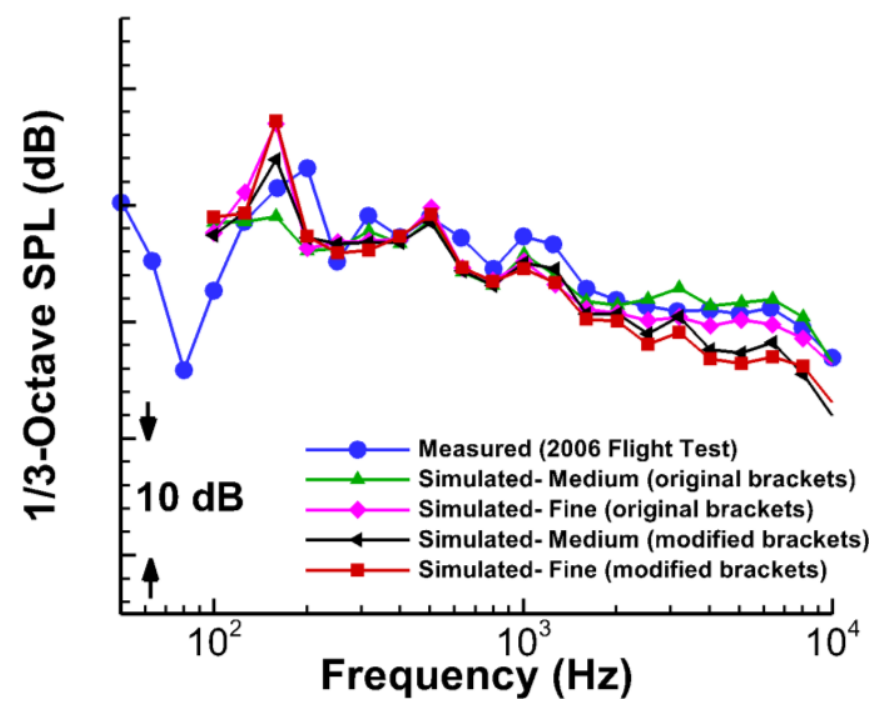

Figure 12. Farfield noise spectra for landing configuration with $39^{\circ}$ flap deflection and main gear retracted. Computed spectra are based on FWH approach using pressure data on aircraft solid surfaces.

Farfield SPL spectra for the case of retracted flap $\left(0^{\circ}\right.$ deflection $)$ with main gear deployed are shown in Fig. 13. In general, very good agreement between predicted and measured sound levels is observed in the frequency range between $600 \mathrm{~Hz}$ and $4 \mathrm{kHz}$. Major differences appear in the very low- and high-frequency segments of the spectrum. At low frequencies, the predicted spectra display a more pronounced tonal behavior with significantly over-predicted peak levels. These tones are associated with the main gear cavity, which extends across the lower part of the fuselage and connects the left and right wheel wells. As is common with most computational approaches, the present simulations over-predict the levels for the cavity tones and their imprints on the farfield noise. This is usually a consequence of the numerical simulation representing the cavity as perfectly rigid without any wall compliance, absorption or leakage effects. At high frequencies, the simulations significantly under-predict the measured data. The under-prediction of high frequency noise observed in Figure 13 for both medium and fine resolutions is, most likely, a result of insufficient resolution of various main gear finer structures (e.g., brake assembly, hydraulic lines, etc.). Again, we 
note that the high frequency content of the measured spectrum is affected by possibly large uncertainties caused by variability in local atmospheric conditions and background noise during the flight test. Such effects disproportionately impact the measurement of high-frequency sounds. Better insight on the simulated high-frequency noise trends would have required the use of an even finer grid. The computational cost and resources necessary for such an endeavor would be prohibitively high.

Because the configuration with flaps deflected $39^{\circ}$ and main gear deployed is the closest to a complete aircraft in landing attempted, it was also the most computationally challenging. Farfield SPL spectra for this configuration are shown in Fig. 14. Good correspondence between medium and fine resolution results was obtained, indicating that spatial resolution effects were adequately addressed even at the medium resolution level and that, as expected, any lingering effects were mostly confined to the higher frequency range. Also, the agreement between predicted and measured SPLs was rather good for frequencies up to $3 \mathrm{kHz}$. Beyond this frequency, differences emerged due to the increasing effect of bracket noise at higher frequencies. Modification of the brackets seemed to improve agreement with measured levels at higher frequencies. Unfortunately, the latter simulation could not be repeated on the finest grid available before conclusion of the ERA project to determine whether the close agreement attained could be extended to the highest frequencies of interest.

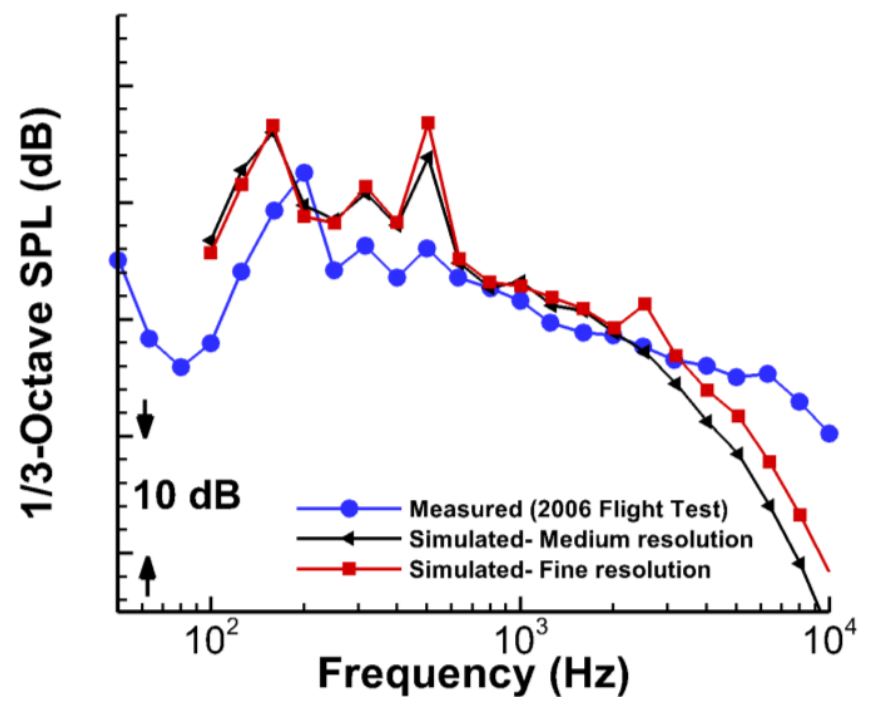

Figure 13. Farfield noise spectra for configuration with flap retracted $\left(0^{\circ}\right)$ and main gear deployed. Computed spectra are based on FWH approach using pressure data on aircraft solid surfaces.

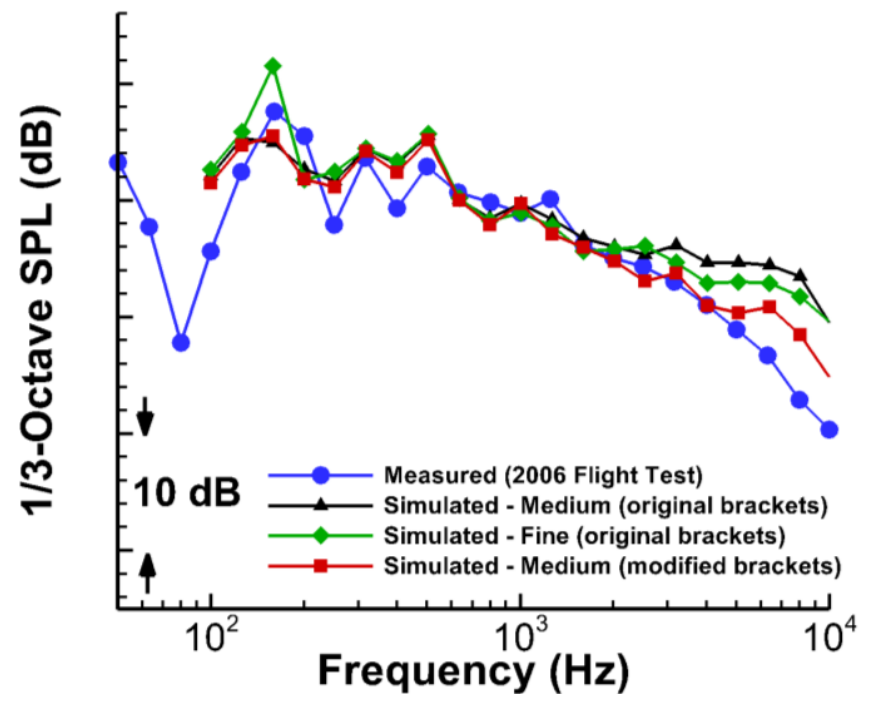

Figure 14. Farfield noise spectra for landing configuration with $39^{\circ}$ flap deflection and main gear deployed. Computed spectra are based on FWH approach using pressure data on aircraft solid surfaces. 


\section{E. Source Localization Maps}

Simulation results were also validated using source localization (beamform) contour maps. Sample maps at various frequencies for the configuration with flaps deflected $39^{\circ}$, main gear deployed, and modified flap brackets are presented in Fig. 15. The contours have been normalized so that the peak SPL level in each map is zero. The axes give the vehicle dimensions in feet. Both the experimentally measured flight-test data ${ }^{18}$ and the simulated results used the same non-uniform spiral cluster of 167 microphones that was deployed at Wallops Flight Facility for the flight test. To generate the maps from the computed data, the synthetic pressure record at each microphone location within the array was constructed using the pressures on the solid surfaces of the aircraft in a FWH propagation code. The 2006 flight test data was processed with a standard delay and sum beamforming approach ${ }^{18}$ in the time domain. Similarly, the synthetic pressure (microphone) records were processed with a standard beamforming algorithm, but in the frequency domain. . In the flight test, the microphone data obtained for aircraft positions of 100 feet in front of the center of the array to directly over the center were used in the processing. This time segment was chosen to minimize the influence of engine noise that becomes more significant after the aircraft passes. For the simulations, the array is effectively directly under the aircraft for all time. Figure 15a compares the contour maps from simulated and measured data for a frequency of $879 \mathrm{~Hz}$. Very good agreement is observed, with both maps highlighting the flap tips and main landing gear as the prominent sound sources at this frequency. Due to limits in array resolution and the proximity of the main gear to the flap inboard edge, it was not possible to separate gear noise from the noise generated at the flap tip. Contour maps at $1,270 \mathrm{~Hz}$ are presented in Fig. 15b. Good correspondence between simulated and measured data was achieved for this frequency as well. The flap outboard edge is the dominant noise source, and subsequent windtunnel testing ${ }^{19}$ revealed the cause to be an interaction between the tip vortex and the bulb-seal cavity flow. At this frequency, residual engine noise in the flight data is most likely causing the high levels in the region directly downstream of the wheels. Contour maps at $3,288 \mathrm{~Hz}$ are shown in Fig. 15c. At this frequency, significant differences emerge between the contours obtained from simulated and measured data. While the contribution from the outboard flap tips is still visible in the measured map, the dominant source seems to be engine noise radiating from the inlet, with possible contributions from the gear and flap inboard tip. Note, however, that it is not possible to identify the dominant source with certainty. In contrast, the simulated contour map clearly identifies the outboard tips as the dominant sources at 3,288 Hz, with the flap brackets and main gear acting as secondary sources. The fine resolution simulation for this configuration was not completed by the end of the ERA project, and is unavailable for this paper. Hence, the possible effects of increased spatial resolution on maps at this and higher frequencies are currently unknown. Realizing that the maps from the flight data correspond to a moving aircraft (source) with residual engine noise and uncontrolled meteorological conditions, whereas the present simulations are tailored to a stationary source without propulsion noise under controlled conditions, the overall agreement obtained at low to moderate frequencies is quite remarkable and very auspicious for the utility of future simulations.
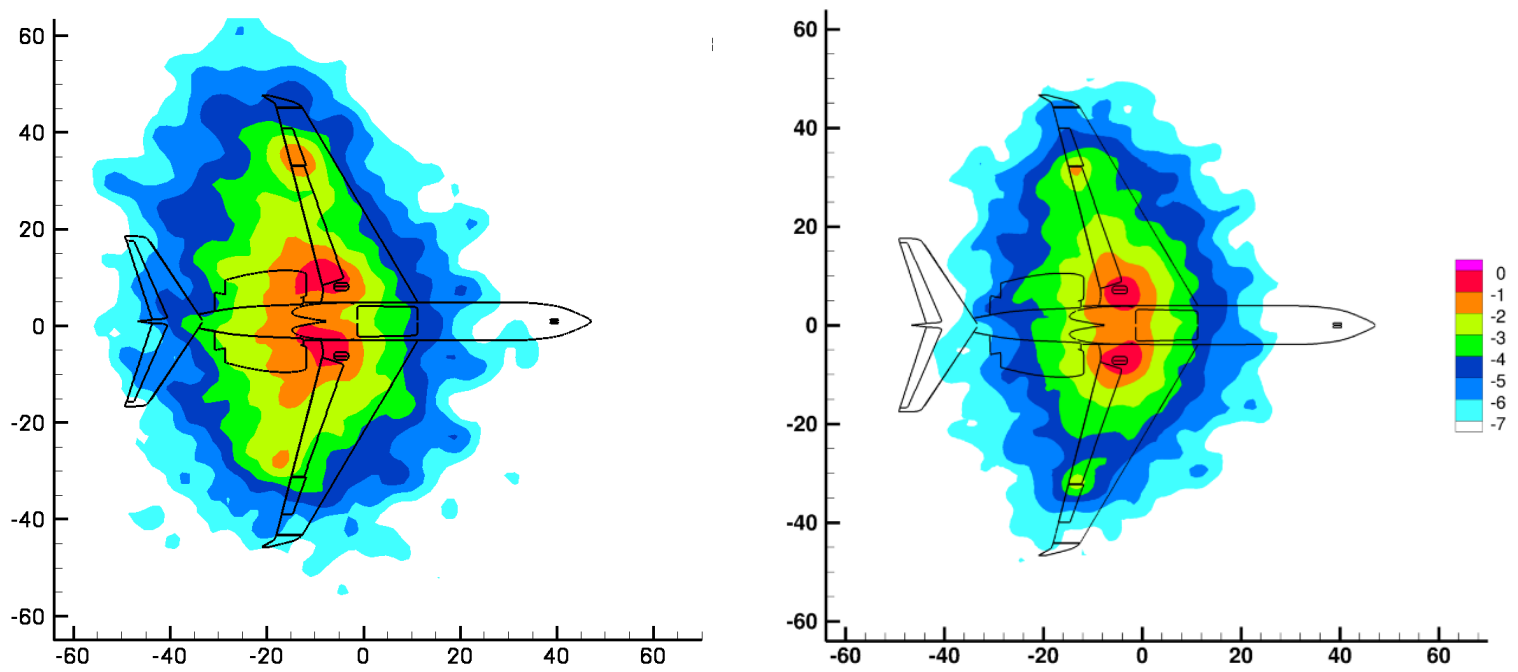

a) $879 \mathrm{~Hz}$, left image: flight test, right image: simulation.

Figure 15. Source localization (beamform) contour maps of SPL (in dB down from peak) for landing configuration with $39^{\circ}$ flap deflection, main gear deployed, and modified brackets (continued). 

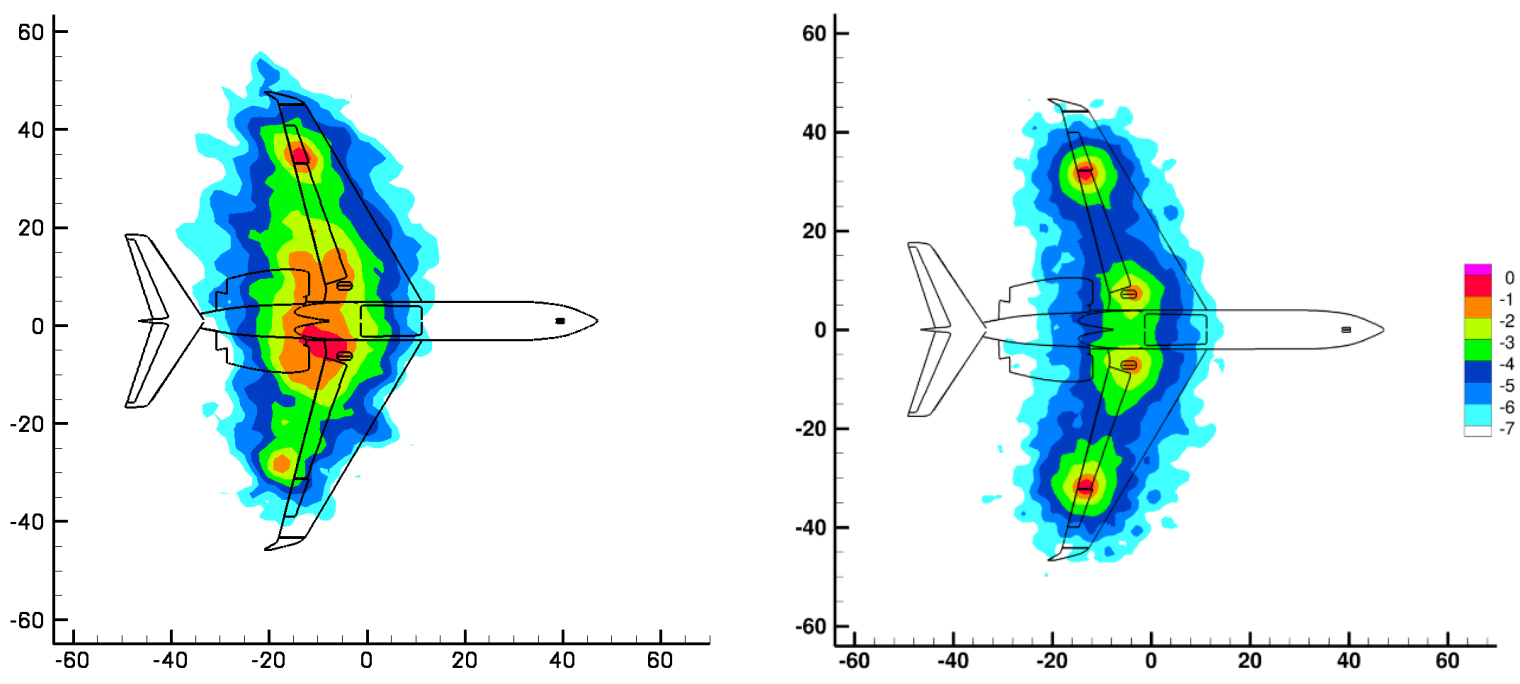

b) $1,270 \mathrm{~Hz}$, left image: flight test, right image: simulation
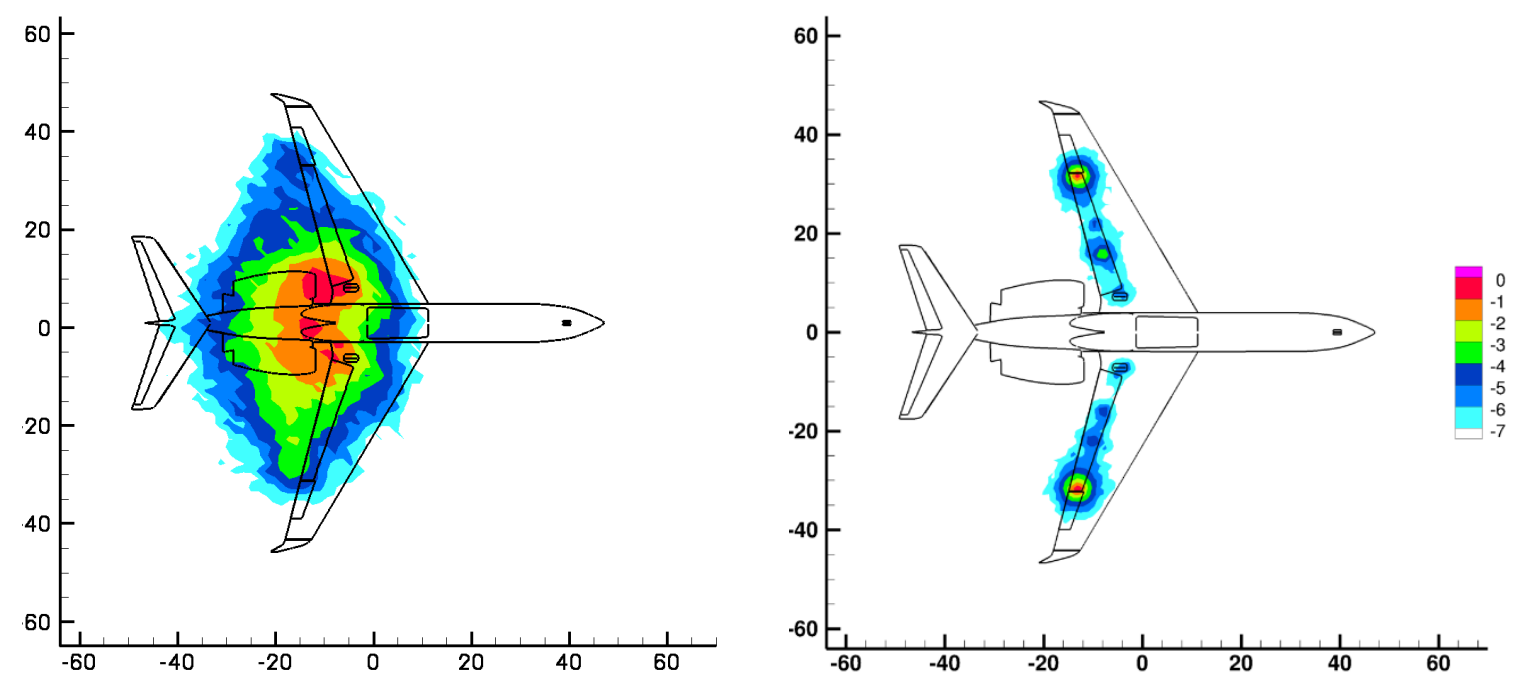

c) $3,288 \mathrm{~Hz}$, left image: flight test, right image: simulation.

Figure 15. Concluded.

The present full-scale computations are the result of a first-time attempt to simulate the unsteady flow around a complete aircraft with high geometrical complexity. The success of the computational simulations resulted in a set of validated solutions that fostered the subsequent full-scale evaluation of several flap and main gear noise reduction concepts developed under the ERA project. The results of that effort are discussed in a companion paper by Khorrami et al. ${ }^{17}$

\section{Concluding Remarks}

The present paper documents an extensive computational study aimed at advancing simulation-based airframe noise prediction to include a full-scale, complete aircraft in landing configuration. To achieve this ambitious goal, the computational effort was centered on conducting high-fidelity, time-accurate simulations of a full-scale Gulfstream aircraft that features nearly all of the finer details for the flap and main landing gear components.

The Exa Corporation PowerFLOW ${ }^{\circledR}$ lattice Boltzmann solver was used to perform the computations for free-air at a Mach number of 0.2 and a Re of 10.5 million, which is roughly half the flight Re (based on full-scale wing mean aerodynamic chord and aircraft landing speed). To ascertain solution convergence, the majority of the simulations were performed on three successively finer grids. Sample steady and unsteady surface pressures on the flap, in particular near the side edges, were used to demonstrate the degree of grid independence that was achieved. The results 
obtained from the full-scale simulations were validated using measured and predicted surface pressures of an 18\%scale version of the same aircraft, properly corrected for size. This approach was necessary because fully vetted flight test data were not available. Overall, good correspondence between model- and full-scale surface pressures was observed. The most noticeable differences occurred at the flap outboard tip and were caused by a combination of Re effects and increased geometrical complexities of the tip cavity and bulb seal assembly.

Farfield noise data from a 2006 flight test, acquired for three baseline configurations (flaps deflected $39^{\circ}$ with and without main landing gear deployed, and retracted flap with main gear deployed), were used to validate the computed solutions on medium and fine spatial resolution grids. With simulated spectra appropriately corrected to compensate for ground reflection effects present in the 2006 flight measurements, the broadband component of the computed farfield noise is in very good agreement with measured data for all three aircraft configurations considered. Most of the differences occurred at relatively high frequencies and were associated with higher uncertainties in the measured spectra and contributions from flap bracket noise. At low frequencies, the simulated spectra for configurations with gear deployed possessed a strong tonal component associated with the gear cavity. Although present in the measured spectra, these cavity tones had relatively low amplitudes. Sample source localization maps obtained from the simulated data for the configuration with flaps deflected $39^{\circ}$, main gear deployed, and modified flap brackets were shown to be in good agreement with corresponding maps from the 2006 flight test at low to moderate frequencies.

The full-scale computations presented here are the result of a first-time attempt to simulate the unsteady flow around a complete aircraft with high geometrical complexity. The success of the simulations promoted the subsequent computational evaluation of several full-scale flap and main gear noise reduction concepts developed under the ERA project. The results of that effort are summarized in a companion paper.

\section{Acknowledgments}

This work was supported by the ERA project under the Integrated Aviation Systems Program (IASP) of NASA. Special thanks are due to Thomas Van de Ven (retired) and Scott Dutton of GAC for facilitating and assisting with transfer and development of the full-scale aircraft geometry model. Our gratitude also goes to Scott Brynildsen of Vigyan, Inc. for providing geometry modifications and CAD support. We would also like to express our sincere appreciation to Patrick Moran of the NASA Ames Research Center for high-quality visualizations and animations of the large data sets. The authors are also grateful to Benjamin Duda and Jason Appelbaum of Exa Corporation for their support on post-processing and geometry preparation. All the simulations were performed on the Pleiades supercomputer at the NASA Advanced Supercomputing (NAS) facility at Ames Research Center. The logistical support provided by NAS staff, in particular Yan-Tyng (Sherry) Chang of Computer Sciences Corporation, is greatly appreciated.

\section{References}

${ }^{1}$ Dobrzynski, W., "Almost 40 Years of Airframe Noise Research: What Did We Achieve," J. Aircraft, Vol. 47, No. 2, March-April 2010, pp. 353-367.

${ }^{2}$ Michel, U., Barsikow, B., Helbig, J., Hellmig, M., and Schüttpelz, M., "Flyover Noise Measurements on Landing Aircraft with a Microphone Array," AIAA Paper 98-2336, May 1998.

${ }^{3}$ Piet, J.-F., Elias, G., and Lebigot, P., "Localization of Acoustic Sources from a Landing Aircraft with a Microphone Array," AIAA Paper 99-1811, May 1999.

${ }^{4}$ Stoker, R., Guo, Y., Streett, C. L., and Burnside, N., "Airframe Noise Source Locations of a 777 Aircraft in Flight and Comparisons with Past Model Tests," AIAA Paper 2003-3232, May 2003.

${ }^{5}$ Brusniak, L., Underbrink, J. R., and Stoker, R. W., "Acoustic Imaging of Aircraft Noise Sources Using Large Aperture Phased Arrays," AIAA Paper 2006-2715, May 2006.

${ }^{6}$ Elkoby, R., Brusniak, L., Stoker, R., Khorrami, M. R., Abeysinghe, A., and Moe, J. W., "Airframe Noise Results from the QTD II Flight Test Program,” AIAA Paper 2007-3457, May 2007.

${ }^{7}$ Li. Y., Satti, R., Lew, P.-T., Shock, R., and Nölting, S., "Computational Aeroacoustic Analysis of Flow Around a Complex Nose Landing Gear Configuration," AIAA Paper 2008-2916, May 2008.

${ }^{8}$ Keating, A., Dethioux, P., Satti, R., Nölting, S., Louis, J., Van de Ven, T., and Vieito, R., "Computational Aeroacoustics Validation and Analysis of a Nose Landing Gear," AIAA Paper 2009-3154, May 2009.

${ }^{9}$ Fares, E. and Nölting, S., "Unsteady Flow Simulation of a High-Lift Configuration using a Lattice-Boltzmann Approach," AIAA Paper 2011-0869, January 2011.

${ }^{10}$ Vatsa, V., Lockard, D. P., Khorrami, M. R., and Carlson, J.-R., "Aeroacoustic Simulation of a Nose Landing Gear in an Open-Jet Facility using FUN3D," AIAA Paper 2012-2280, June 2012. 
${ }^{11}$ Casalino, D., Nölting, S., Fares, E., Vand de Ven, T., Perot, F., and Bres, G., "Towards Numerical Aircraft Noise Certification: Analysis of a Full-Scale Landing Gear in Fly-Over Configuration," AIAA Paper 2012-2235, June 2012.

${ }^{12}$ Khorrami, M. R. and Mineck, R. E., "Towards Full Aircraft Airframe Noise Prediction: Detached Eddy Simulations," AIAA Paper 2014-2480, June 2014.

${ }^{13}$ Khorrami, M. R., Fares, E., and Casalino, D., "Towards Full-Aircraft Airframe Noise Prediction: Lattice-Boltzmann Simulations," AIAA Paper 2014-2481, June 2014.

${ }^{14}$ Fares, E., Casalino, D., and Khorrami, M. R., "Evaluation of Airframe Noise Reduction Concepts via Simulations using a Lattice-Boltzmann Approach,” AIAA Paper 2015-2988, June 2015.

${ }^{15}$ Khorrami, M. R., Mineck, R. E., Yao, C. S., and Jenkins, L. N., “A Comparative Study of Simulated and measured Gear-Flap Flow Interaction,” AIAA Paper 2015-2989, June 2015.

${ }^{16}$ Fares, E., Duda, B., and Khorrami, M. R., "Airframe Noise Prediction of a Full Aircraft in Model and Full Scale Using a Lattice Boltzmann Approach," Paper to be presented at the $22^{\text {nd }}$ AIAA/CEAS Aeroacoustics Conference in Lyon, France, May-June 2016.

${ }^{17}$ Khorrami, M. R., Fares, E., Duda, B., and Hazir, A., “Computational Evaluation of Airframe Noise Reduction Concepts at Full Scale," Paper to be presented at the $22^{\text {nd }}$ AIAA/CEAS Aeroacoustics Conference in Lyon, France, May-June 2016

${ }^{18}$ Khorrami, M. R., Lockard, D. P., Humphreys, Jr., W. M., Choudhari, M. M., and Van de Ven, T., "Preliminary Analysis of Acoustic Measurements from the NASA-Gulfstream Airframe Noise Flight Test," AIAA Paper 20082814, May 2008.

${ }^{19}$ Khorrami, M. R., Humphreys, Jr., W. M., Lockard, D. P., and Ravetta, P. A., “Aeroacoustic Evaluation of Flap and Landing Gear Reduction Concepts," AIAA Paper 2014-2478, 2014.

${ }^{20}$ Chen, H., Chen, S., and Matthaeus, W., "Recovery of the Navier-Stokes Equations Using a Lattice-Gas Boltzmann Method," Physical Review A, Vol. 45, No. 8, 1992, pp. 5339-5342.

${ }^{21}$ Lockard, D., "Summary of the Tandem Cylinder Solutions from the Benchmark Problems for Airframe Noise Computations-I Workshop," AIAA Paper 2011-0353, 2011.

${ }^{22}$ Yakhot, V. and Orszag, S. A., "Renormalization Group Analysis of Turbulence. I. Basic Theory," J. Sci. Comput., Vol. 1, No. 2, 1986, pp. 3-51.

${ }^{23}$ Chen, H., Kandasamy, S., Orszag, S. A., Succi, S., and Yakhot, V., "Extended Bolzmann Kinetic Equation for Turbulent Flows," Science, Vol. 301, No. 5633, 2003, pp. 633-636.

${ }^{24}$ Chen J., "Volumetric formulation of the lattice Boltzmann method for fluid dynamics: Basic concept," Physical Review E, Vol. 58, No. 3, 1998, pp. 3955-3963.

${ }^{25}$ Chen, H., Teixeira, C., and Molvig, K., "Realization of Fluid Boundary Conditions via Discrete Boltzmann Dynamics," International Journal of Modern Physics C, Vol. 9, No. 8, 1998, pp. 1281-1292.

${ }^{26}$ Ffowcs Williams, J. E. and Hawkings, D. L., "Sound Generated by Turbulence and Surfaces in Arbitrary Motion," Philosophical Transactions of the Royal Society, Vol. A264, No. 1151, 1969, pp. 321-342.

${ }^{27}$ Farassat, F. and Succi, G. P., "The Prediction of Helicopter Discrete Frequency Noise,” Vertica, Vol. 7, No. 4, 1983, pp. 309-320.

${ }^{28}$ Najafi-Yazdi, A., Brès, G. A., and Mongeau, L., “An Acoustic Analogy Formulation for Moving Sources in Uniformly Moving Media,” Proceeding of The Royal Society of London A, Vol. 467 (2125), 2011, pp. 144-165.

${ }^{29}$ Khorrami, M. R., Hannon, J. A., Neuhart, D. H., Markowski, G. A., and Van de Ven, T., “Aeroacoustic Studies of a High-Fidelity Aircraft Model: Part 1-Steady Aerodynamic Measurements,” AIAA Paper 2012-2233, June 2012.

${ }^{30}$ Khorrami, M. R. and Neuhart, D. H., “Aeroacoustic Studies of a High-Fidelity Aircraft Model: Part 2- Unsteady Surface Pressures," AIAA Paper 2012-2234, June 2012. 